\title{
Quantificação de Componentes Monocíclicos da Antracnose do Feijoeiro*
}

\author{
Maristella Dalla Pria', Lilian Amorim² \& Armando Bergamin Filho \\ ${ }^{1}$ Departamento de Fitotecnia e Fitossanidade, Universidade Estadual de Ponta Grossa, Praça Santos Andrade s/n, \\ CEP 84010-790, Ponta Grossa, PR, fax: (042) 220-3072, email: mdallapria@uepg.br; ²Departamento de Entomologia, Fitopatologia \\ e Zoologia Agrícola, Escola Superior de Agricultura “Luiz de Queiroz”, Cx. Postal 09, CEP 13418-900, Piracicaba, SP
}

(Aceito para publicação em 09/04/2003)

Autor para correspondência: Maristella Dalla Pria

DALlA PRIA, M., AMORIM, L. \& BERGAMIN FILHO, A. Quantificação de componentes monocíclicos da antracnose do feijoeiro. Fitopatologia Brasileira 28:401-407. 2003.

\section{RESUMO}

Foram realizados experimentos em ambiente controlado, em câmaras de crescimento, para a antracnose do feijoeiro (Phaseolus vulgaris) causada por Colletotrichum lindemuthianum, em duas cultivares, Rosinha e Carioca, para a quantificação de componentes monocíclicos. Os componentes monocíclicos avaliados foram período de incubação e severidade da doença, sob as temperaturas de $6,10,14,17,21,25,29$ e $33^{\circ} \mathrm{C}$. A antracnose não se desenvolveu nas temperaturas de 6 e $33^{\circ} \mathrm{C}$. A severidade foi mais elevada a $17{ }^{\circ} \mathrm{C}$ e o menor período de incubação ocorreu a $29{ }^{\circ} \mathrm{C}$, para a cv. Rosinha, e $25{ }^{\circ} \mathrm{C}$ para a cv. Carioca. A função beta generalizada mostrou bom ajuste aos dados de temperatura. $\mathrm{O}$ efeito do período de molhamento foliar $(5,6,12,18,24,30,36$, 42, 48 e 54 h) na severidade da doença também foi avaliado. Não houve incremento na severidade da antracnose do feijoeiro para períodos de molhamento superiores a $24 \mathrm{~h}$. As variações de severidade da antracnose do feijoeiro, em relação ao número de horas de molhamento foliar, foram explicadas pelo modelo de Richards. A função BetaRichards explicou o efeito combinado da temperatura e duração do molhamento foliar na severidade da antracnose do feijoeiro para ambas as cultivares.

Palavras-chave adicionais: Phaseolus vulgaris, temperatura, duração molhamento foliar.

\section{ABSTRACT}

Quantification of monocyclic components of the common bean anthracnose

Monocyclic components of anthracnose caused by Colletotrichum lindemuthianum were quantified on two bean (Phaseolus vulgaris) cultivars Rosinha and Carioca, in growth chambers. Incubation period and disease severity were assessed, at temperatures of $6,10,14,17,21,25,29$ and $33{ }^{\circ} \mathrm{C}$. Anthracnose did not develop at temperatures of 6 and $33{ }^{\circ} \mathrm{C}$. The severity was highest at $17{ }^{\circ} \mathrm{C}$ and the shortest incubation periods occurred at $29{ }^{\circ} \mathrm{C}$ for the cv. Rosinha and $25^{\circ} \mathrm{C}$ for the cv. Carioca. Beta function was fitted to data of disease severity at different temperatures. The effect of the leaf wetness duration $(5,6,12,18,24,30,36,42,48$ and $54 \mathrm{~h})$ in disease severity was assessed as well. There was no increment in anthracnose severity of the bean for periods of wetness superior to $24 \mathrm{~h}$. The variations of anthracnose in relation to the number of hours of leaf wetness were explained by the Richards model. The Beta-Richards function explained the combined effect of the temperature and leaf wetness duration in the severity of anthracnose, for both varieties.

\section{INTRODUÇÃO}

A antracnose, causada por Colletotrichum lindemuthianum (Sacc \& Magn.) Lams.-Scrib., é considerada uma das doenças mais graves da cultura do feijoeiro (Phaseolus vulgaris L.) no Brasil, uma vez que pode ocorrer em toda parte aérea da planta e ao encontrar condições favoráveis, causar grandes danos na produção (Zambolim \& Chaves, 1978). Esta doença é de distribuição ampla, já tendo sido constatada em vários países da Europa, África, Ásia e América. No Brasil, ocorre nos Estados do Rio Grande do Sul, Santa Catarina, Paraná, São Paulo, Minas Gerais, Bahia, Pernambuco, Espírito Santo, Alagoas, Sergipe e Paraíba. Esta

\footnotetext{
* Parte da tese de doutorado do primeiro autor, ESALQ/USP (1997). financiada pelo CNPq.
}

ampla abrangência deve-se ao uso de cultivares suscetíveis em regiões onde ocorrem temperaturas amenas, variando de 13 a $26^{\circ} \mathrm{C}$, em determinadas épocas do ano, e alta umidade, podendo causar redução na produção de até $100 \%$. Isto pode ocorrer principalmente quando sementes contaminadas são usadas para plantio e quando ocorrem períodos prolongados de condições favoráveis ao desenvolvimento da doença (Resende, 1989; Schwartz, 1994).

Os danos são maiores quanto mais precoce for o aparecimento da doença na lavoura. Além de diminuir o rendimento da cultura, a antracnose deprecia a qualidade do produto por ocasionar manchas nos grãos, tornando-os impróprios para o consumo (Sartorato \& Rava, 1994). A antracnose afeta plantas de feijão em todos os estádios de crescimento, provocando lesões em folhas, caule, ramos, vagens e sementes (Sartorato \& Rava, 1994). 


\section{Dalla Pria et al.}

A descrição do processo de desenvolvimento e a quantificação das doenças de plantas são os principais objetivos da epidemiologia. Estudos epidemiológicos são baseados em experimentos de campo, experimentos em câmaras de crescimento sob condições controladas e modelos de simulação (Kranz, 1974; Aust \& Kranz, 1988). Resultados de experimentos realizados em ambientes controlados fornecem uma aproximação do comportamento das doenças nas condições naturais de epidemias. Nesses experimentos, avalia-se o monociclo por meio de seus componentes. Parlevliet (1979) definiu os componentes monocíclicos mais utilizados nos estudos epidemiológicos: período de incubação (período de tempo compreendido entre a inoculação e o aparecimento dos sintomas); período latente (período de tempo entre a inoculação e a produção de esporos); freqüência de infecção (número de lesões produzidas por unidade de área, a partir de uma concentração conhecida de inóculo); esporulação (número de esporos produzidos por unidade de área de tecido afetado e/ou por unidade de tempo). A quantificação do período de incubação e do período latente tem sido feita, experimentalmente, considerando-se o período de tempo entre a inoculação e o aparecimento de 50\% dos sintomas ou lesões esporulantes, respectivamente (Parlevliet, 1975).

Quanto maiores os períodos de incubação e latente e quanto menor a freqüência de infecção, menor será o número de ciclos do patógeno sobre seu hospedeiro. Conseqüentemente, menor será a severidade final da doença (Parlevliet, 1975). Assim, determinando-se o efeito do ambiente sobre os componentes monocíclicos do patossistema, pode-se inferir sobre as condições ambientais favoráveis ao desenvolvimento de epidemias.

Todos os componentes monicíclicos sofrem influência do ambiente. A grandeza de cada um deles é relativa às condições ambientais sob as quais o sistema esteve exposto. Assim, para compreender o sistema em estudo é necessário quantificar os componentes monicíclicos sob diferentes condições do ambiente.

O objetivo deste trabalho foi avaliar os efeitos da temperatura e do período de molhamento sobre os componentes monocíclicos da antracnose, em plantas de feijoeiro mantidas sob condições de ambiente controlado.

\section{MATERIALEMÉTODOS}

\section{Avaliação do efeito da temperatura nos componentes monocíclicos da antracnose}

Foram utilizadas duas cultivares de feijoeiro, Rosinha (altamente suscetível à antracnose) e Carioca (moderadamente suscetível à antracnose) (Rava \& Sartorato, 1994). As duas cultivares foram semeadas em vasos de alumínio contendo 2,5 1 de solo esterilizado, mantidos em casa de vegetação. Cinco dias após a emergência das plântulas, foi realizado o desbaste mantendo-se duas plantas por vaso. Quarenta e oito horas antes da inoculação, os vasos foram transferidos para câmaras de crescimento (marca CONVIRON modelo E7), com temperatura e fotoperíodo previamente regulados, para aclimatação das plantas.

O inóculo de $C$. lindemuthianum foi produzido em tubos contendo o meio de vagem inteira - MVI (Dalla Pria et al., 1997). Os tubos com as culturas fúngicas foram incubados por 15 dias à temperatura de $22^{\circ} \mathrm{C} \pm 2$, no escuro.

A suspensão de conídios foi obtida de culturas incubadas por um período de 15 dias, mantidas a $22^{\circ} \mathrm{C} \pm 2$, no escuro. Os tubos com as colônias fúngicas foram lavados com água destilada, acrescida de Tween 20 (uma gota para cada $100 \mathrm{ml}$ ), com o auxílio de um pincel de cerdas macias, para auxiliar na liberação dos conídios. A suspensão de esporos foi calibrada para 2,0 × $10^{6}$ conídios $/ \mathrm{ml}$, préestabelecida por meio de testes preliminares. As plantas foram inoculadas quando a primeira folha trifoliolada encontravase completamente expandida, aproximadamente 20 dias após o plantio.

A superfície superior e a inferior das duas primeiras folhas trifolioladas foram atomizadas com a suspensão de inóculo até o ponto de escorrimento. Após a inoculação, as plantas permaneceram em câmara úmida, dentro de câmaras de crescimento, com fotoperíodo de $12 \mathrm{~h}$ de luz. Plantas do tratamento testemunha foram atomizadas com água destilada + Tween 20 (uma gota para cada $100 \mathrm{ml}$ de água). As temperaturas avaliadas nesse ensaio foram: 6, 10, 14, 17, 21, 25,29 e $33^{\circ} \mathrm{C}$, e os períodos de molhamento foliar utilizados foram: $30,36,42 \mathrm{e} 48 \mathrm{~h}$.

O delineamento experimental foi o inteiramente casualizado, no esquema fatorial, com dois fatores (temperatura e umidade), com quatro repetições. Cada parcela experimental foi constituída de um vaso, com duas plantas, onde duas folhas trifolioladas por planta foram avaliadas.

Avaliou-se a severidade da doença por meio de escala diagramática (Figura 1). As observações foram realizadas diariamente até o surgimento dos primeiros sintomas. A partir daí, as avaliações seguiram a intervalos de 12 ou 24 h, dependendo da velocidade de crescimento da doença, e finalizaram quando a porcentagem de tecido atacado não apresentava aumento. Com a média dos dados de severidade das quatro repetições, foram obtidas as curvas de progresso da doença para cada uma das temperaturas testadas em função de dias após a inoculação. As curvas foram ajustadas ao modelo de Richards (Bergamin Filho, 1995):

$$
\begin{aligned}
& \mathrm{Y}=\mathrm{K}(1-(\beta) \exp (-\mathrm{RX}))^{(1 /(1-\mathrm{M}))}, \text { quando } \mathrm{M}<1 \\
& \mathrm{Y}=\mathrm{K}(1+(\beta) \exp (-\mathrm{RX}))^{(1 /(1-\mathrm{M}))}, \text { quando } \mathrm{M}>1
\end{aligned}
$$

onde $\mathrm{Y}$ é a severidade da doença, $\mathrm{X}$ é o tempo, $\mathrm{K}$ representa a assíntota do modelo, $\beta$ representa a constante de integração, $\mathrm{R}$, a taxa de progresso da doença e $\mathrm{M}$ é o parâmetro que representa a forma da curva (Schuh \& Adamowicz, 1993). Para o ajuste dos dados foi utilizado o programa STATISTICA for Windows versão 4.5 (StatSoft, Tulsa, EUA).

A partir das curvas de progresso da doença e dos valores das assíntotas $(\mathrm{K})$ determinados para cada uma das 
temperaturas foi calculado o período de incubação, tempo para que a severidade se iguale a $\mathrm{K} / 2$, ou seja, período compreendido entre a inoculação e o surgimento de $50 \%$ dos sintomas.

Com os parâmetros calculados e valores de $\mathrm{K}$ determinados, foi possível estabelecer uma relação entre a severidade da antracnose e a temperatura. Os pontos foram ajustados por meio de regressão não-linear, usando-se a função Beta generalizada (Hau \& Kranz, 1990),

$$
\mathrm{Y}=\mathrm{B}_{1}\left(\left(\mathrm{X}-\mathrm{B}_{2}\right)^{\mathrm{B} 4}\right)\left(\left(\mathrm{B}_{3}-\mathrm{X}\right)^{\mathrm{B} 5}\right)
$$

onde os parâmetros $\mathrm{B}_{2}$ e $\mathrm{B}_{3}$ representam a temperatura mínima e máxima, respectivamente, $Y$ representa a severidade, $X$ representa a temperatura, os parâmetros $\mathrm{B}_{1}, \mathrm{~B}_{4}$ e $\mathrm{B}_{5}$ são parâmetros do próprio modelo e não possuem significado biológico. Para o ajuste dos dados de temperatura para a antracnose foi necessário fixar os valores de $\mathrm{B}_{2}$ (temperatura mínima) em $6^{\circ} \mathrm{C}$ e $\mathrm{B}_{3}$ (temperatura máxima) em $33^{\circ} \mathrm{C}$, uma vez que nestas temperaturas não foram constatados quaisquer sintomas da doença.

\section{Avaliação do efeito do período de molhamento foliar nos componentes monocíclicos da antracnose}

Após o estabelecimento da temperatura ótima para o desenvolvimento da doença, foram realizados experimentos para determinação do efeito da duração do período de molhamento foliar sobre os componentes monocíclicos da antracnose do feijoeiro. Os períodos de câmara úmida testados foram: 5, 6, 12, 18, 24, 30, 36, 42, 48 e $54 \mathrm{~h}$, sob temperatura constante de $21^{\circ} \mathrm{C}$.

A produção das mudas, preparo do inóculo, inoculação e avaliação das plantas foram feitos como descrito no item anterior. O delineamento experimental foi inteiramente casualizado, com quatro repetições. Cada parcela experimental era constituída de um vaso, com duas plantas, onde foram avaliadas duas folhas trifolioladas por planta.

Com a média dos dados de severidade para cada um dos períodos de molhamento foliar testados foram estabelecidas as curvas de progresso da doença, considerandose a porcentagem de tecido doente em função de dias após a inoculação. As curvas foram ajustadas por meio de regressão não-linear, utilizando-se o modelo de Richards. Com os dados obtidos foi possível elaborar um gráfico da severidade das doenças em função da duração do molhamento foliar.

O efeito combinado da temperatura e da duração do molhamento foliar na severidade da doença foi descrito pelo modelo Beta-Richards:

$$
\mathrm{Z}=\mathrm{B}_{1}\left(\left(\mathrm{X}-\mathrm{B}_{2}\right)^{\mathrm{B} 4}\right)\left(\left(\mathrm{B}_{3}-\mathrm{X}\right)^{\mathrm{B} 5}\left(1 \pm(\beta) \exp \left(-\mathrm{B}_{6} \mathrm{Y}\right)\right)^{\mathrm{B} 7}\right.
$$

e possibilitou o cálculo da superfície resposta. Os parâmetros foram ajustados por meio de regressão não-linear dos dados, onde: $\mathrm{Z}=$ severidade da doença $(\%), \mathrm{X}=$ temperatura $\left({ }^{\circ} \mathrm{C}\right), \mathrm{Y}$ $=$ duração do molhamento foliar (horas), $\beta=$ constante de integração, $\mathrm{B}_{2}=$ temperatura mínima, $\mathrm{B}_{3}=$ temperatura máxima, $\mathrm{B}_{7}=$ forma da curva, $\mathrm{B}_{1}, \mathrm{~B}_{4}, \mathrm{~B}_{5}$ e $\mathrm{B}_{6}=$ parâmetros do modelo.

\section{RESULTADOS E DISCUSSÃO}

\section{Avaliação do efeito da temperatura nos componentes monocíclicos da antracnose}

A temperatura mostrou grande influência sobre a severidade e sobre o período de incubação da antracnose do feijoeiro. As equações que apresentaram melhor ajuste para os dados de severidade da antracnose em função da temperatura, com os respectivos coeficientes de determinação, encontram-se na Tabela 1.

O efeito da temperatura sobre a severidade da antracnose do feijoeiro foi explicado pela função Beta generalizada com coeficiente de determinação de 0,93 para a cv. Carioca (Figura 2A) e 0,77 para a cv. Rosinha (Figura 2B). Esta função também foi utilizada por Bacchi (1993) para ajuste do período latente e freqüência de infecção para a ferrugem do feijoeiro causada por Uromyces appendiculatus (Pers.) Unger, em função da temperatura. Através do ajuste dos dados as duas cultivares testadas tiveram comportamento semelhante em relação à temperatura, ficando o ótimo a $17^{\circ} \mathrm{C}$ (Figura 2). A temperatura mínima para o desenvolvimento da antracnose foi de $6^{\circ} \mathrm{C}$ para as duas cultivares, e a máxima, de $33^{\circ} \mathrm{C}$.

Trabalhos executados com outras espécies de Colletotrichum mostraram resultados semelhantes. Dillard (1988), em estudos realizados in vitro, constatou que a máxima germinação de conídios de Colletotrichum coccodes (Wallr.) Hughes ocorreu a $22{ }^{\circ} \mathrm{C}$ não havendo germinação a $7{ }^{\circ} \mathrm{C}$. Menos de $70 \%$ dos conídios germinaram na temperatura de 10 ou $31^{\circ} \mathrm{C}$. A temperatura ótima encontrada por Monroe $e t$

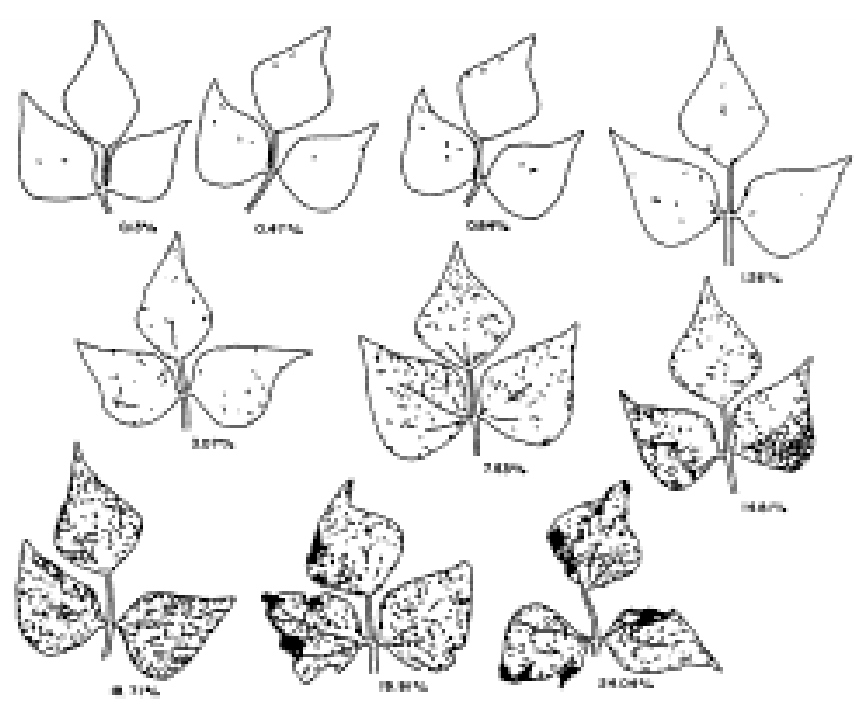

FIG. 1 - Escala diagramática de severidade (porcentagem de área foliar afetada) da antracnose causada por Colletotrichum lindemuthianum do feijoeiro (Phaseolus vulgaris) (Carneiro et al., não publicado). 
TABELA 1 - Equações e respectivos coeficientes de determinação $\left(\mathrm{R}^{2}\right)$, obtidos via regressões não-lineares, para ajuste dos dados de severidade da antracnose causada por Colletotrichum lindemuthianum do feijoeiro (Phaseolus vulgaris), em função do tempo, para cada uma das temperaturas testadas, para as cultivares Carioca e Rosinha

\begin{tabular}{|c|c|}
\hline Equação & $\mathbf{R}^{2}$ \\
\hline \multicolumn{2}{|l|}{ Cultivar Carioca } \\
\hline $\left.\left.\operatorname{SEV}_{6}{ }^{\circ}{ }^{\circ}=(0,38)(1-(3,77) \exp (-(0,054) \mathrm{T}))^{(1 /(1-(0,9994)}\right)\right)$ & 0,960 \\
\hline $\left.\left.\operatorname{SEV}_{10}{ }^{\circ}{ }^{\circ}=(15,80)(1-(2,13) \exp (-(0,020) \mathrm{T}))^{(1 /(1-(0,9958)}\right)\right)$ & 0,996 \\
\hline $\left.\left.\operatorname{SEV}_{14}{ }^{\circ} C^{\circ}=(19,87)(1+(16,94) \exp (-(0,064) T))^{(1 /(1-(1,0004)}\right)\right)$ & 0,997 \\
\hline $\left.\left.\mathrm{SEV}_{17}{ }^{\circ} \mathrm{C}=(23,27)(1-(66,53) \exp (-(0,103) \mathrm{T}))^{(1 /(1-(0,9998)}\right)\right)$ & 0,992 \\
\hline $\left.\left.\mathrm{SEV}_{21}{ }^{\circ} \mathrm{C}=(20,65)(1+(1037,2) \exp (-(0,066) \mathrm{T}))^{(1 /(1-(1,3224)}\right)\right)$ & 0,998 \\
\hline $\left.\left.\operatorname{SEV}_{25^{\circ} \mathrm{C}}=(3,63)(1+(1,24) \exp (-(0,061) \mathrm{T}))^{(1 /(1-(1,0006)}\right)\right)$ & 0,988 \\
\hline $\left.\left.\mathrm{SEV}_{29}{ }^{\circ} \mathrm{C}=(0,70)(1-(9,89) \exp (-(0,053) \mathrm{T}))^{(1 /(1-(0,9981)}\right)\right)$ & 0,980 \\
\hline \multicolumn{2}{|l|}{ Cultivar Rosinha } \\
\hline $\left.\left.\operatorname{SEV}_{6}{ }^{\circ} C^{\circ}=(0,07)(1-(2,20) \exp (-(0,067) \mathrm{T}))^{(1 /(1-(0,9997)}\right)\right)$ & 0,999 \\
\hline $\left.\left.\operatorname{SEV}_{10}{ }^{\circ} \mathrm{C}=(10,55)(1-(11,40) \exp (-(0,029) \mathrm{T}))^{(1 /(1-(0,9972)}\right)\right)$ & 0,997 \\
\hline $\left.\left.\operatorname{SEV}_{14}{ }^{\circ} C^{\circ}=(18,47)(1-(0,64) \exp (-(0,025) \mathrm{T}))^{(1 /(1-(0,9990)}\right)\right)$ & 0,991 \\
\hline $\left.\left.\operatorname{SEV}_{17}{ }^{\circ} \mathrm{C}=(22,88)(1-(5,58) \exp (-(0,050) \mathrm{T}))^{(1 /(1-(0,9993)}\right)\right)$ & 0,994 \\
\hline $\left.\left.\operatorname{SEV}_{21}{ }^{\circ} \mathrm{C}=(22,61)(1-(6,86) \exp (-(0,051) \mathrm{T}))^{(1 /(1-(0,9993)}\right)\right)$ & 0,993 \\
\hline 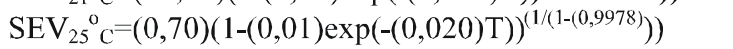 & 0,850 \\
\hline $\left.\left.\mathrm{SEV}_{29}{ }^{\circ} \mathrm{C}=(0,29)(1-(0,22) \exp (-(0,046) \mathrm{T}))^{(1 /(1-(0,9936)}\right)\right)$ & 0,980 \\
\hline
\end{tabular}

$\mathrm{Sev}=$ severidade $(\%), \mathrm{T}=$ tempo (horas)

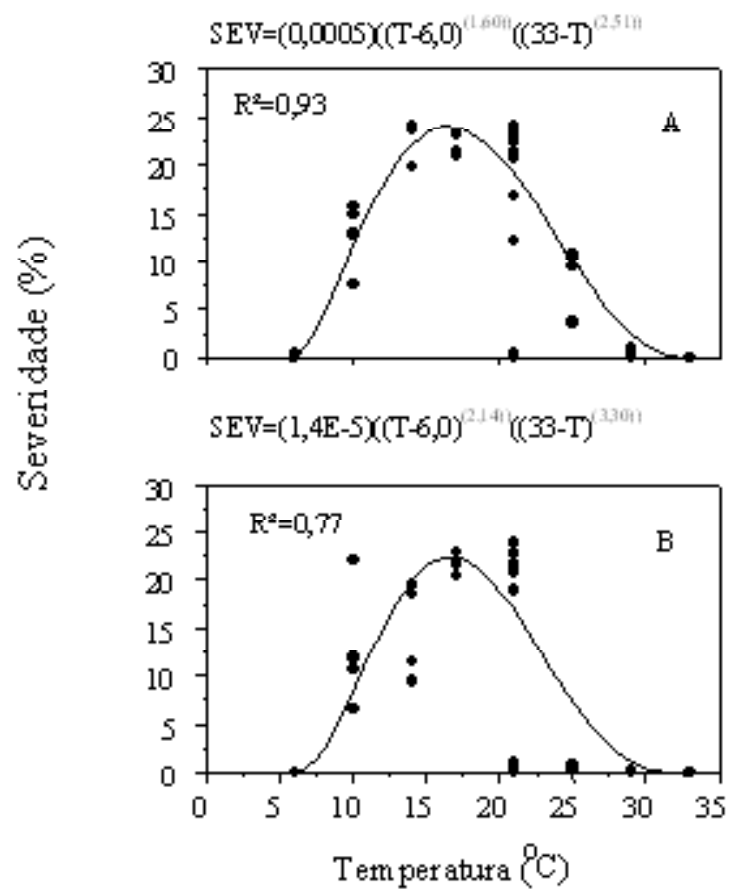

FIG. 2 - Severidade da antracnose causada por Colletotrichum lindemuthianum do feijoeiro (Phaseolus vulgaris), em diferentes temperaturas, nas cultivares Carioca (A) e Rosinha (B). Cada ponto representa a média de quatro repetições. Linhas representam o ajuste do modelo Beta-generalizado

al. (1997) para a infecção de Colletotrichum orbiculare (Berk. \& Mont.) (Arx) em melancia [Citrullus lanatus (Thumb) Matsumi \& Nakai], ficou entre 21 e $24^{\circ} \mathrm{C}$, um pouco acima da encontrada para C. lindemuthianum, que ficou em $17^{\circ} \mathrm{C}$. Estes autores também verificaram que a intensidade de doença foi elevada na temperatura de $21^{\circ} \mathrm{C}$ e que nas temperaturas mais baixas $\left(12 \mathrm{e} 15^{\circ} \mathrm{C}\right)$ e na temperatura mais alta $30^{\circ} \mathrm{C}$, a quantidade de tecido infectado foi mínima.

Estudos realizados por Rahe \& Kuc (1970) mostraram que o número e o tamanho das lesões da antracnose do feijoeiro diminuem com o aumento da temperatura de incubação numa faixa de 28 a $32{ }^{\circ} \mathrm{C}$, e nenhuma lesão se desenvolveu em plantas mantidas sob temperaturas acima de $32{ }^{\circ} \mathrm{C}$. Em condições de campo, no Canadá, altas temperaturas reduzem a severidade da antracnose do feijoeiro, pois $C$. lindemuthianum é sensível a altas temperaturas, sendo seu crescimento reprimido em temperatura entre $30-31^{\circ} \mathrm{C}$. No sul do Canadá as temperaturas no campo freqüentemente atingem níveis entre $25-35^{\circ} \mathrm{C}$, e ainda a doença continua a se manifestar. Isto, provavelmente, deve-se ao fato das baixas temperaturas noturnas estarem moderando o efeito das altas temperaturas diurnas, fornecendo condições ambientais favoráveis à infecção e desenvolvimento da doença.

O presente trabalho confirmou os resultados obtidos por Tu \& Aylesworth (1980) onde não ocorreu infecção por C. lindemuthianum quando plantas inoculadas foram incubadas em ambiente úmido a temperaturas acima de $28^{\circ} \mathrm{C}$. Outros autores também constataram que a temperatura limitante para a infecção encontra-se na faixa de 26,6 a $32^{\circ} \mathrm{C}$ (Martinez Salazar \& Andersen, 1957; Zaumeyer \& Thomas, 1957).

Após a obtenção das curvas de progresso da antracnose em função de diferentes temperaturas, foi possível quantificar o período de incubação (Figura 3), explicado pela função Beta generalizada, com coeficiente de determinação de 0,94 para a cv. Carioca (Figura 3A) e 0,90 para a cv. Rosinha (Figura 3B). O período de incubação diminuiu com o aumento da temperatura até $25^{\circ} \mathrm{C}$ para a cv. Carioca e até $29^{\circ} \mathrm{C}$ para a cv. Rosinha. Para a cv. Rosinha, o período de incubação foi menor que para a cv. Carioca, nas faixas de temperatura entre 21 e $29{ }^{\circ} \mathrm{C}$. Os primeiros sintomas surgiram $48 \mathrm{~h}$ após a inoculação para todas as temperaturas testadas.

Resultados contrastantes foram observados por $\mathrm{Tu}$ (1982), que verificou que o aumento na temperatura diária promoveu um alongamento no período de incubação e diminuiu levemente a severidade da antracnose do feijoeiro. Os sintomas não se desenvolveram quando as plantas inoculadas foram mantidas em temperatura constante de $28 \mathrm{e}$ $32^{\circ} \mathrm{C}$.

\section{Avaliação do efeito do período de molhamento nos componentes monocíclicos da antracnose}

O efeito da duração do período de molhamento foliar sobre a antracnose do feijoeiro foi semelhante nas duas cultivares testadas (Figuras 4A e 4B). Períodos de molhamento foliar entre 18 e $24 \mathrm{~h}$ proporcionaram acentuado aumento de tecido doente. Já períodos superiores a 24 h de molhamento foliar não redundaram em aumento de severidade da doença. Os primeiros sintomas da antracnose surgiram após $6 \mathrm{~h}$ de 
$\mathrm{PI}=(1376,22)(\mathrm{T}-(7,31))^{(-165)}\left(((30,73)-\mathrm{T})^{(1,2314}\right.$

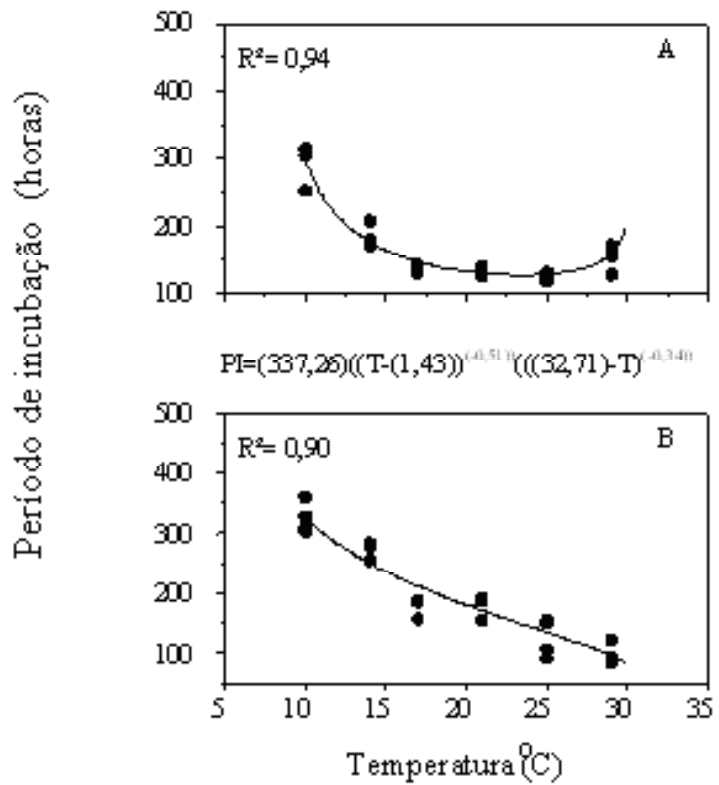

FIG. 3 - Efeito da temperatura no período de incubação da antracnose do do feijoeiro (Phaseolus vulgaris) causada por Colletotrichum lindemuthianum, nas cultivares Carioca (A) e Rosinha (B). Cada ponto representa a média de quatro repetições.

molhamento foliar. Os dados obtidos foram ajustados pelo modelo de Richards, com coeficiente de determinação de 0,97 e 0,99 para as cultivares Carioca e Rosinha, respectivamente.

Neste trabalho foi possível a determinação do período mínimo de umidade para a infecção por $C$. lindemuthianum, pois com $6 \mathrm{~h}$ de molhamento foliar foram observados os sintomas da antracnose e com $5 \mathrm{~h}$ não houve desenvolvimento de lesões.

Para C. lindemuthianum, a combinação dos dados referentes à temperatura e duração do molhamento foliar com a severidade da antracnose do feijoeiro, permitiu calcular a superfície resposta, que foi ajustada pela função BetaRichards: sev $\left.=(0,0005 \text { (temp-6 })^{1,61}(33 \text {-temp })^{2,52}\right)(1-11,3 \exp (-$ $0,72 \mathrm{~mol}))^{17985}$, onde sev é a severidade em porcentagem, temp, a temperatura em ${ }^{\circ} \mathrm{C}$ e mol o período de molhamento em horas, com coeficiente de determinação de 0,94 para a cv. Carioca $\left(\right.$ Figura 5) e sev $=\left(0,00004(\text { temp- } 5,44)^{2,04}(33 \text {-temp })^{2,96}\right)(1$ $54,5 \exp (-0,68 \mathrm{~mol}))^{7734,4}, \mathrm{com} \mathrm{R}{ }^{2}=0,78$ para a cv. Rosinha (Figura 6). A máxima severidade ocorreu na faixa de 17 a $21^{\circ} \mathrm{C}$ e $24 \mathrm{~h}$ de molhamento, para ambas as cultivares.

Muitos estudos têm investigado o papel do aumento do período de molhamento na ocorrência das doenças, estabelecendo que a duração do período de molhamento necessário para o estabelecimento das doenças depende da temperatura. Os trabalhos de Evans et al. (1992), mostraram que o molhamento foliar determina a quantidade de esporos que germinam e a penetração nos tecidos do hospedeiro e a temperatura determina a velocidade e a extensão da infecção (Lalancette et al., 1988; Evans et al., 1992).

Tanto para Puccinia hordei Otth. em plantas de cevada

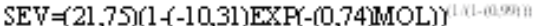

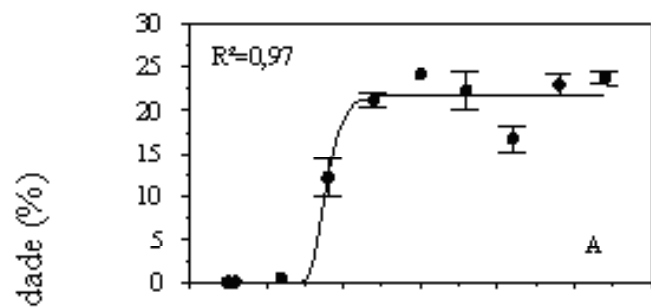

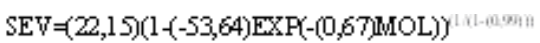

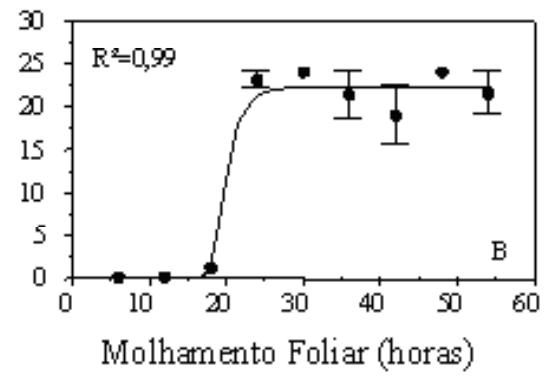

FIG. 4 - Severidade da antracnose do feijoeiro (Phaseolus vulgaris), causada por Colletotrichum lindemuthianum, em diferentes períodos de molhamento foliar, nas cultivares Carioca (A) e Rosinha (B). Cada ponto representa a média de quatro repetições. Barras representam o erro padrão da média.

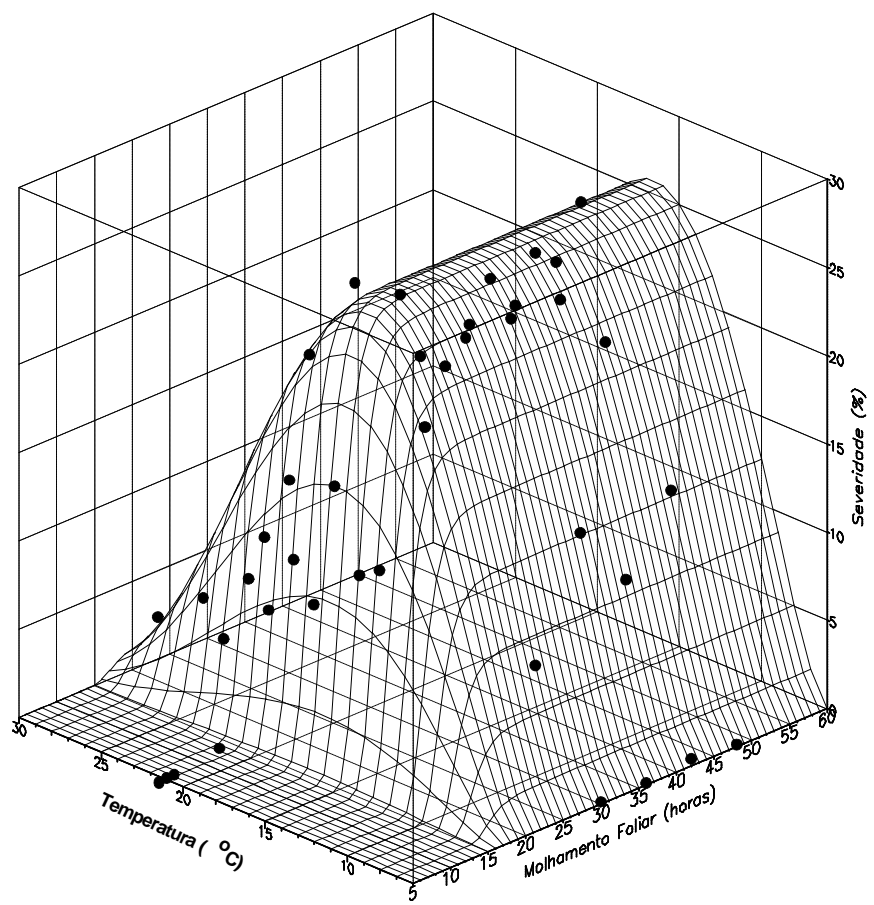

FIG. 5 - Efeito do período de molhamento foliar e da temperatura na severidade da antracnose do feijoeiro (Phaseolus vulgaris) causada por Colletotrichum lindemuthianum na cultivar Carioca.

(Hordeum vulgare L.) como para Puccinia arachidis Speg. em plantas de amendoim (Arachis hypogaea $\mathrm{L}$.), o principal efeito da temperatura foi no período mínimo de molhamento 


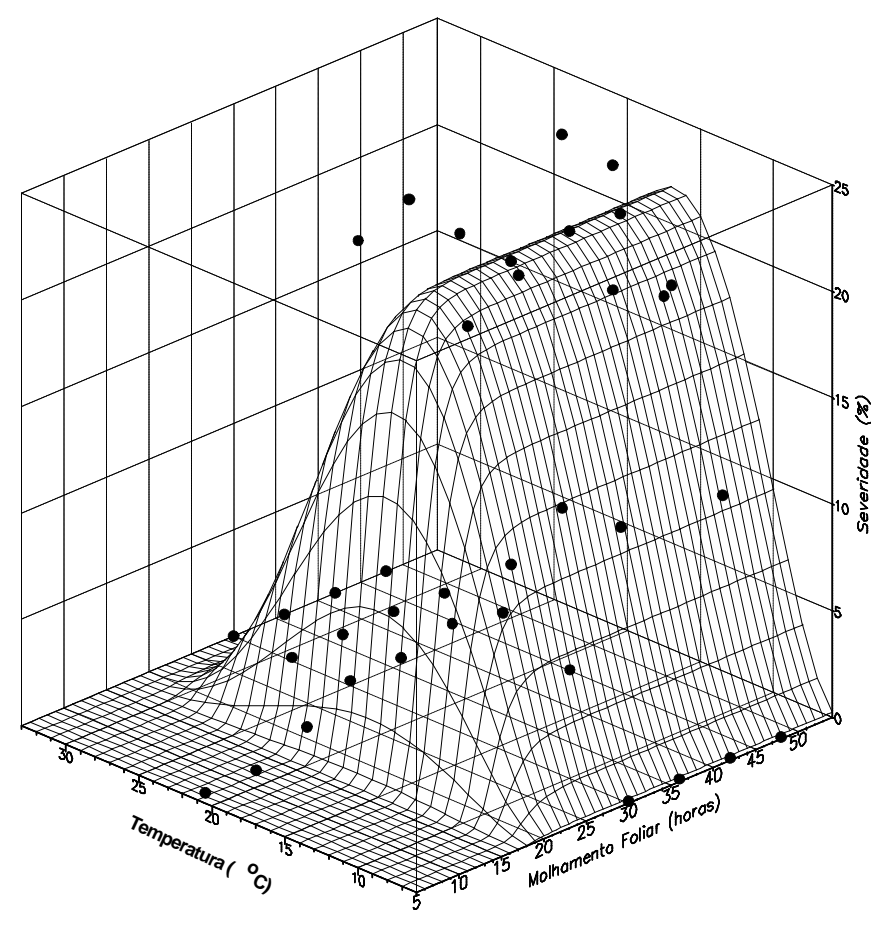

FIG. 6 - Efeito do período de molhamento foliar e da temperatura na severidade da antracnose do feijoeiro (Phaseolus vulgaris) causada por Colletotrichum lindemuthianum na cultivar Rosinha.

foliar exigido para que a infecção iniciasse e no número máximo de lesões. Aparentemente é necessário que o molhamento foliar seja contínuo durante todo o processo de inoculação. Quando folhas de amendoim são secas por período de $1 \mathrm{~h}$ e então reumedecidas e mantidas por $30 \mathrm{~h} \mathrm{em}$ temperatura ótima, poucas lesões se desenvolvem (Butler \& Jadhav, 1991). As curvas encontradas por estes autores para explicar o efeito da temperatura e do período de molhamento sobre a densidade de lesões, tiveram formato semelhante àquelas obtidas neste trabalho para $C$. lindemuthianum.

\section{REFERÊNCIAS BIBLIOGRÁFICAS}

AUST, H.J. \& KRANZ, J. Experiments and procedures in epidemiological field studies. In: Kranz, J. \& Rotem, J. (Eds.). Experimental techniques in plant disease epidemiology. Berlin: SpringerVerlag, 1988. pp.7-17.

BACCHI, L.M.A. Quantificação de parâmetros monocíclicos relacionada a epidemias no sistema Uromyces appendiculatus - feijoeiro. (Tese de Doutorado). Piracicaba. Universidade de São Paulo. 1993.

BERGAMIN FILHO, A. Curvas de progresso da doença. In: Bergamin Filho, A., Kimati, H. \& Amorim, L. (Eds.) Manual de Fitopatologia: princípios e conceitos. São Paulo: Editora Ceres, 1995. v.1, pp.602626.

BUTLER, D.R. \& JADHAV, D.R. Requirements of leaf wetness and temperature for infection of groundnut by rust. Plant Pathology 40:395400. 1991.

DALLA PRIA, M., BERGAMIN FILHO, A. \& AMORIM, L. Avaliação de diferentes meios de cultura na esporulação de
Colletotrichum lindemuthianum, Phaeoisariopsis griseola e Alternaria sp. Summa Phytopathologica 23:188-191. 1997.

DILLARD, H.R. Influence of temperature, $\mathrm{pH}$, osmotic potencial and fungicide sensitivity on germination of conidia and growth from sclerotia of Colletotrichum coccodes in vitro. Phytopathology 78:1357-1361. 1988.

EVANS, K.J., NYQUIST, W.E. \& LATIN, R.X. A model based on temperature and leaf wetness duration for establishment of Alternaria leaf blight of muskmelon. Phytopathology 82:890-895. 1992.

HAU, B. \& KRANZ, J. Mathematics and statistics for analyses in epidemiology. In: Kranz, J. (Ed.) Epidemics of plant disease: mathematics analyses and modeling. Berlin: Springer-Verlag, 1990. pp.12-52.

KRANZ, J. Epidemiology concepts and scope. In: Raychauduri, S.P. \& Verma, F.P. (Eds.). Current trends in plant pathology. Lucknow: University Botany Department, 1974. pp.26-32.

LALANCETTE, N., ELLIS, M.A. \& MADDEN, L.V. Development of an infection efficiency model for Plasmopara viticola on American grape based on temperature and duration leaf wetness. Phytopathology 78:794-800. 1988.

MARTINEZ SALAZAR, I. \& ANDERSEN, A.I. Effects of temperature on spore germination and host infectivity by three strains of Colletotrichum lindemuthianum. Phytopathology 47:23. 1957.

MONROE, J.S., SANTINI, J.B. \& LATIN, R. A model defining the relationship between temperature and leaf wetness duration, and infection of watermelon by Colletotrichum orbiculare. Plant Disease 81:739-742. 1997.

PARLEVLIET, J.E. Partial resistance of barley of leaf rust. Puccinia hordei I. Effect of cultivar and development stage on latent period. Euphytica 24:21-27. 1975.

PARLEVLIET, J.E. Components of resistance that reduce the rate of epidemic development. Annual Review of Phytopathology 17:203222. 1979.

RAHE, J.E. \& KUC, J. Metabolic nature of the infection-limiting effect of heat on bean anthracnose. Phytopathology 60:1005-1009. 1970.

RAVA, C.A. \& SARTORATO, A. Antracnose. In: Sartorato, A. \& Rava, C.A. (Eds.). Principais doenças do feijoero comum e seu controle. Brasília: EMBRAPA,SPI, 1994. pp.17-39.

RESENDE, M.A.V. Seleção de progênies de feijoeiro resistente a Colletotrichum lindemuthianum (Sacc. et Magn) Scrib. na população Esal 501 x To. (Dissertação de Mestrado). Lavras. Universidade Federal de Lavras. 1989.

SARTORATO, A. \& RAVA, C.A. Mancha angular. In: Sartorato, A. \& Rava, C.A. (Eds.). Principais doenças do feijoero comum e seu controle. Brasília: EMBRAPA,SPI, 1994. pp.41-68.

SCHUH, W. \& ADAMOWICZ, A. Influence of assessment time and modeling approach on the relationships between temperature-leaf wetness periods and disease parameters of Septoria glycines on soybean. Phytopathology 83:941-948. 1993.

SCHWARTZ, H.F. Anthracnose. In: Hall, R. (Ed.) Compendium of bean diseases. St. Paul: APS Press, 1994. pp.16-17.

TU, J.C. Effect of temperature on incidence and severity of anthracnose on white bean. Plant Disease 66:781-783. 1982.

TU, J.C. \& AYLESWORTH, J.W. An effective method of screening white (pea) bean seedlings (Phaseolus vulgaris L.) for resistance to Colletotrichum lindemuthianum. Phytopathologische Zeitschrift 
Quantificação de componentes monocíclicos da antracnose (Colletotrichum lindemuthianum)...

99:131-137. 1980.

ZAMBOLIM, L. \& CHAVES, G.M. Doenças do feijoeiro e seu controle. Informe Agropecuário 4:50-63. 1978.
ZAUMEYER, W.J. \& THOMAS, H.R. A monographic study of bean diseases and methods for their control. Washington: USDA, Technical Bulletin, 868. 1957. 